\title{
EQUIVARIANT COMPLETION
}

\section{J. P. MAY}

The purpose of this sequel to [5] is to construct and characterize completions of $G$-spaces, where $G$ is a compact Lie group. We assume the notations and results of [5]. It is a pleasure to thank Pete Bousfield for the proof of Lemma 12.

Since completions are less generally familiar than localizations, a quick summary of the relevant part of the nonequivariant theory is in order. We are interested in spaces not satisfying any finiteness conditions, and we understand completions in the sense of Bousfield and Kan [1] rather than of Sullivan [6]. Most of the following material is implicit or explicit in [1]; the rest is proven in [4], which gives an elementary homotopical account of the theory.

Fix a set of primes $T$. For an Abelian group $A$, define

$$
H_{T} A=\operatorname{Hom}\left(Z\left[T^{-1}\right] / Z, A\right) \quad \text { and } \quad E_{T} A=\operatorname{Ext}\left(Z\left[T^{-1}\right] / Z, A\right) .
$$

Observe that a short exact sequence $0 \rightarrow A^{\prime} \rightarrow A \rightarrow A^{\prime \prime} \rightarrow 0$ gives rise to a long exact sequence

$$
0 \rightarrow H_{T} A^{\prime} \rightarrow H_{T} A \rightarrow H_{T} A^{\prime \prime} \rightarrow E_{T} A^{\prime} \rightarrow E_{T} A \rightarrow E_{T} A^{\prime \prime} \rightarrow 0
$$

By the definition, we have a connecting homomorphism

$$
\delta: A=\operatorname{Hom}(Z, A) \rightarrow E_{T} A .
$$

When $H_{T} A=0, \delta$ is called the completion of $A$ at $T ; A$ is said to be $T$-complete if $H_{T} A=0$ and $\delta$ is an isomorphism. In particular, $H_{T} A$ and $E_{T} A$ are themselves $T$-complete for any $A$.

Here $Z\left[T^{-1}\right] / Z \cong Q / Z_{T}$ is the direct sum over $p \in T$ of the groups $Z_{p^{x}}=\lim _{\rightarrow} Z_{p^{n}}$. Write $H_{p}$ and $E_{p}$ for the functors $H_{T}$ and $E_{T}$ when $T=\{p\}$. Then

$$
H_{T} A=\prod_{p \in T} H_{p} A \quad \text { and } \quad E_{T} A=\prod_{p \in T} E_{p} A
$$

To understand the structure of $E_{T} A$; define

$$
\hat{A_{p}}=\lim _{\leftarrow} A / p^{n} A \quad \text { and } \quad \hat{A}_{T}=\prod_{p \in T} \hat{A}_{p} .
$$

Then $\hat{A}_{p}$ is $p$-complete and there is a natural short exact sequence

$$
0 \rightarrow \underset{\leftarrow}{\lim ^{1} \operatorname{Hom}}\left(Z_{p^{n}}, A\right) \rightarrow E_{p} A \rightarrow \hat{A}_{p} \rightarrow 0
$$

In particular, $H_{T} A=0$ and $E_{T} A \simeq \hat{A}_{T}$ if the $p$-torsion elements of $A$ are of bounded

Received 15 June, 1981; revised 12 August, 1981.

Bull. London Math. Soc., 14 (1982), 231-237 
order for $p \in T$, for example if $A$ is a finitely generated or torsion free $Z_{s}$-module for any set of primes $S$ containing $T$.

Ignoring the topology, any $T$-profinite Abelian group is $T$-complete, but not conversely. The collection of $T$-complete Abelian groups can be characterized as the largest collection for which the following assertion is true. We write $Z_{p}$ for $Z / p Z$.

Proposition 1. The following conditions on a map $f: X \rightarrow Y$ are equivalent; when they hold, $f$ is said to be a $\hat{T}$-cohomology isomorphism.

(i) $f_{*}: H_{*}\left(X ; Z_{p}\right) \rightarrow H_{*}\left(Y ; Z_{p}\right)$ is an isomorphism for all $p \in T$.

(ii) $f^{*}: H^{*}(Y ; A) \rightarrow H^{*}(X ; A)$ is an isomorphism for all $Z_{p}$-vector spaces $A$ and all $p \in T$.

(iii) $f^{*}: H^{*}(Y ; A) \rightarrow H^{*}(X ; A)$ is an isomorphism for all $T$-complete Abelian groups $A$.

The functors $H_{T}$ and $E_{T}$ and the natural transformation $\delta$ can be extended to nilpotent groups. They continue to enjoy properties similar to those in the Abelian case, such as the analogs of (2) and (4). The notions of $T$-completion and of $T$-completeness extend in the obvious way.

THEOREM 2. The following conditions on a nilpotent space $Z$ are equivalent; when they hold, $Z$ is said to be T-complete.

(i) Each group $\pi_{n} Z$ is $T$-complete.

(ii) If $f: X \rightarrow Y$ is a $\hat{T}$-cohomology isomorphism, then

is a bijection.

$$
f^{*}:[Y, Z] \rightarrow[X, Z]
$$

The corresponding assertion for $T$-local spaces is much stronger because one can add the third equivalent condition that the integral homology of $Z$ is $T$-local.

THEOREM 3. Let $X$ be a nilpotent space. The following conditions on a map $\gamma: X \rightarrow \hat{X}_{T}$ from $X$ to a $T$-complete space are equivalent. There exists one and, up to isomorphism in $H \mathscr{T}$, only one map $\gamma$ satisfying these conditions; it is called the completion of $X$ at $T$.

(i) $\gamma^{*}:\left[\hat{X}_{T}, Z\right] \rightarrow[X, Z]$ is a bijection for all $T$-complete spaces $Z$.

(ii) $\gamma: X \rightarrow \hat{X}_{T}$ is a $\hat{T}$-cohomology isomorphism.

Moreover, for each $n \geqslant 1$, there is a natural and splittable short exact sequence

$$
0 \rightarrow E_{T} \pi_{n} X \rightarrow \pi_{n} \hat{X}_{T} \rightarrow H_{T} \pi_{n-1} X \rightarrow 0 .
$$

If $H_{T} \pi_{n} X=0$ for all $n$, then $\gamma$ is also characterized by

(iii) Each $\gamma_{*}: \pi_{n} X \rightarrow \pi_{n} \hat{X}_{T}$ is a completion at $T$.

Again, the analog for localizations is stronger by virtue of the fourth equivalent condition that $\lambda$ induces localization at $T$ on integral homology. 
Write $\hat{X}_{p}$ for $\hat{X}_{T}$ when $T=\{p\}$. The universal property (i) implies that $\gamma: X \rightarrow \hat{X}_{p}$ factors through a map $\pi_{p}: \hat{X}_{T} \rightarrow \hat{X}_{p}$ if $p \in T$, and (4) has the following consequence.

Corollary 4. The map $\left(\pi_{p}\right): \hat{X}_{T} \rightarrow \prod_{p \in T} \hat{X}_{p}$ is an equivalence.

Nevertheless, it is natural to consider completions at general sets of primes $T$. Clearly a $T$-cohomology isomorphism is a $\hat{T}$-cohomology isomorphism, and this has the following consequence.

Corollary 5. The completion $\gamma: X \rightarrow \hat{X}_{T}$ is the composite of the localization $\lambda: X \rightarrow X_{T}$ and the completion $\gamma: X_{T} \rightarrow \hat{X}_{T}$.

That is, the completion of $X$ is the same as the completion of $X_{T}$.

We shall prove the equivariant versions of these results.

All of the algebraic definitions and results summarized in and around formulas (1)-(6) extend immediately to coefficient systems $M: \mathcal{O} \rightarrow \mathrm{Ab}$, by composition of functors. Thus $\left(E_{T} M\right)(G / H)=E_{T} M(G / H)$, and so forth. In particular, we say that a coefficient system $M$ is $T$-complete if each $M(G / H)$ is $T$-complete.

We shall need the following simple construction. Let $F$ denote the functor which assigns to an Abelian group $A$ the free Abelian group on its underlying set and let $R A$ denote the kernel of the natural epimorphism $F A \rightarrow A$. The functors $F$ and $R$ extend to coefficient systems, giving a natural short exact sequence

$$
0 \rightarrow R M \stackrel{i}{\rightarrow} F M \stackrel{\pi}{\rightarrow} M \rightarrow 0
$$

(We do not claim, and it is not true, that $F M$ and $R M$ are projective objects of the Abelian category of coefficient systems.)

We begin work by considering the equivariant version of Proposition 1. We can only give part of the proof at this point.

Proposition 6. Consider the following conditions on a G-map $f: X \rightarrow Y$.

(i) Each $f^{H}: X^{H} \rightarrow Y^{H}$ is a $\hat{T}$-cohomology isomorphism.

(ii) $f^{*}: H_{G}^{*}(Y ; M) \rightarrow H_{G}^{*}(X ; M)$ is an isomorphism for all $Z_{p}$-vector space valued coefficient systems $M$ and all $p \in T$.

(iii) $f^{*}: H_{G}^{*}(Y ; M) \rightarrow H_{G}^{*}(X ; M)$ is an isomorphism for all $T$-complete coefficient systems $M$.

Then (i) implies (ii), and (ii) and (iii) are equivalent. If $X$ and $Y$ are G-nilpotent, all three conditions are equivalent.

Proof. Obviously (iii) implies (ii), and (i) implies (ii) by Proposition 1 and the case $R=Z_{p}$ of [5, Proposition 4]. Assume (ii). By the five lemma and the long exact cohomology sequences associated to the exact sequences

$$
0 \rightarrow p^{n+1} M / p^{n} M \rightarrow M / p^{n} M \rightarrow M / p^{n+1} M \rightarrow 0
$$


and to the exact sequence defining the inverse limit $\hat{M}_{p}$, we see that $f^{*}$ is an isomorphism with coefficients in $\hat{M}_{T}$ for any coefficient system $M$. Now let $M$ be $T$-complete. Applying the functor $E_{T}$ to the exact sequence (7) and using (2) and (6), we obtain an exact sequence

$$
0 \rightarrow(R M)_{T} \rightarrow(F M)_{T} \rightarrow M \rightarrow 0
$$

and (iii) follows from the five lemma and the associated long exact cohomology sequence. When $X$ and $Y$ are $G$-nilpotent, Theorem 14 below will imply that both (i) and (iii) are equivalent to the assertion that $f$ induces an equivalence of completions at $T$.

We agree to take condition (iii) as our definition of a $T$-cohomology isomorphism of $G$-spaces.

REMARK 7. The analog for localization theory asserts that if all $f^{H}: X^{H} \rightarrow Y^{H}$ are $T$-cohomology isomorphisms, then $f: X \rightarrow Y$ is a $T$-cohomology isomorphism, and conversely if $X$ and $Y$ are $G$-nilpotent. The proof is immediate from [5, Proposition 4 and Theorem 10].

The following observation is immediate from the first statement of [5, Lemma 5].

LEMMA 8. If $f: X \rightarrow Y$ is a nonequivariant $\hat{T}$-cohomology isomorphism, then $1 \wedge f:(G / H)_{+} \wedge X \rightarrow(G / H)_{+} \wedge Y$ is an equivariant $\hat{T}$-cohomology isomorphism.

Definition 9. A $G$-tower $X$ (as specified in [5, Definition 6]) is $T$-complete if each $M_{n}$ is a $T$-complete coefficient system.

The following analog of [5, Proposition 7] admits precisely the same homotopical proof by induction over cocells and is thus completely elementary; see [4].

Proposition 10. Let $Z$ be a T-complete G-tower. If $f: X \rightarrow Y$ is a $\hat{T}$-cohomology isomorphism, then

is a bijection.

$$
f^{*}:[Y, Z]_{G} \rightarrow[X, Z]_{G}
$$

To exploit this result, we need the following analog of $[\mathbf{5}$, Proposition 8 (iii)].

Proposition 11. If $X$ is a G-nilpotent G-CW complex and each $X^{H}$ is $T$-complete, there is a $T$-complete nilpotent $G$-tower $Y$ and a weak $G$-equivalence $X \rightarrow Y$.

This is clear if $X$ is $G$-simple. As in the proof of [5; Proposition 8], the general case is easily proven by standard arguments once one knows that the functor $\pi_{1} X$ from $\mathcal{C}$ to groups is nilpotent and acts nilpotently on the functor $\pi_{n} X$ for $n \geqslant 2$, with all Abelian subquotients being $T$-complete. This is a consequence of the following result, which is due to Pete Bousfield. 
LemMA 12. (i) There is a functor $\left\{\Gamma_{T}^{k}\right\}$ from $T$-complete nilpotent groups to central series such that $\Gamma_{T}^{1} \pi=\pi$, each Abelian subquotient $\Gamma_{T}^{k} \pi / \Gamma_{T}^{k+1} \pi$ is $T$-complete, and $\left\{\Gamma_{T}^{k} \pi\right\}$ has the same length as the lower central series $\left\{\Gamma^{k} \pi\right\}$.

(ii) There is a functor $\left\{\Gamma_{T}^{k}\right\}$ from $T$-complete modules over groups (both the underlying $T$-complete Abelian Group $A$ and the acting group $\pi$ being allowed to vary) to central series such that $\Gamma_{T}^{1} A=A$, each $\pi$-trivial subquotient $\Gamma_{T}^{k} A / \Gamma_{T}^{k+1} A$ is $T$-complete, and $\left\{\Gamma_{T}^{k} A\right\}$ has the same length as the lower central series $\left\{\Gamma^{k} A\right\}$.

Proof. The point is that the upper central series fails to be functorial and the lower central series $\left\{\Gamma^{k}\right\}$ presumably fails to yield $T$-complete subquotients. For (i), define $\Gamma_{T}^{k} \pi$ to be the kernel of the composite

$$
\pi \rightarrow \pi / \Gamma^{k} \pi \stackrel{\delta}{\rightarrow} E_{T}\left(\pi / \Gamma^{k} \pi\right)
$$

Since $\pi$ is $T$-complete, use of the nilpotent group version of the exact sequence (2), in particular the centrality of the image of $H_{T} A^{\prime \prime}$ in $E_{T} A^{\prime}$, shows that this composite is an epimorphism, $\Gamma_{T}^{k} \pi$ is $T$-complete, and $\Gamma_{T}^{k} \pi / \Gamma_{T}^{k+1} \pi$ is central in $\pi / \Gamma_{T}^{k+1} \pi$. For (ii), note that an action of a group $\pi$ on an Abelian group $A$ extends by functoriality and naturality to an action of $\pi$ on $E_{T} A$ such that $\delta: A \rightarrow E_{T} A$ is a $\pi$-morphism. From here, the argument is the same as for (i).

The equivariant analog of Theorem 2 now follows from Propositions 10 and 11 and Lemma 8, exactly as in the proof of [5, Theorem 9].

THEOREM 13. Let $Z$ be a $G$-nilpotent $G$-space. Then the following conditions are equivalent. When they hold, $Z$ is said to be a T-complete $G$-space.

(i) Each $Z^{H}$ is $T$-complete in the nonequivariant sense.

(ii) If $f: X \rightarrow Y$ is a $\hat{T}$-cohomology isomorphism of $G$-spaces, then

$$
f^{*}:[Y, Z]_{G} \rightarrow[X, Z]_{G}
$$

is a bijection.

This brings us to our main result, the analog of Theorem 3.

THEOREM 14. Let $X$ be a $G$-nilpotent $G$-space. Then the following conditions on a $G$-map $\gamma: X \rightarrow \hat{X}_{T}$ from $X$ to a $T$-complete $G$-space $\hat{X}_{T}$ are equivalent.

(i) $\gamma^{*}:\left[\hat{X}_{T}, Z\right]_{G} \rightarrow[X, Z]_{G}$ is a bijection for all $T$-complete $G$-spaces $Z$.

(ii) $\gamma: X \rightarrow \hat{X}_{T}$ is a $\hat{T}$-cohomology isomorphism.

(iii) Each $\gamma^{H}: X^{H} \rightarrow \hat{X}_{T}^{H}$ is a completion at $T$ in the nonequivariant sense.

There exists one and, up to isomorphism in HGT, only one G-map $\gamma$ satisfying these conditions; it is called the completion of $X$ at $T$.

Corollaries 4 and 5 clearly remain valid as stated in the equivariant case.

Just as in the proof of [5, Theorem 10], the previous theorem and the part of Proposition 6 already proven imply that it suffices to construct a $G$-map $\gamma: X \rightarrow X_{T}$ 
which satisfies (iii). The construction will precisely parallel [5, Construction 12], once completions of spaces $K(M, n)$ are obtained. As in the nonequivariant case, $K(M, n)_{T}$ will itself be an Eilenberg-MacLane $G$-space if and only if $H_{T} M=0$ but will always be a 2 -stage $G$-space.

If $H_{T} M=0$, we apply Elmendorf's functorial construction of spaces $K(M, n)$ in [2] to obtain a $G$-map

$$
\gamma: K(M, n) \rightarrow K\left(E_{T} M, n\right)
$$

from the completion $\delta: M \rightarrow E_{T} M ; \gamma$ is a completion at $T$ since its restrictions

$$
\gamma^{H}: K(M, n)^{H}=K(M(G / H), n) \rightarrow K\left(E_{T} M(G / H), n\right)=K\left(E_{T} M, n\right)^{H}
$$

are completions at $T$ by the last criterion of Theorem 3.

For general $M$, consider the exact sequence (7). Since $H_{T} R M=H_{T} F M=0$, the exact sequence (2) gives an exact sequence of coefficient systems

$$
0 \rightarrow H_{T} M \rightarrow E_{T} R M \stackrel{E_{T} i}{\longrightarrow} E_{T} F M \rightarrow E_{T} M \rightarrow 0 .
$$

Consider the following diagram:

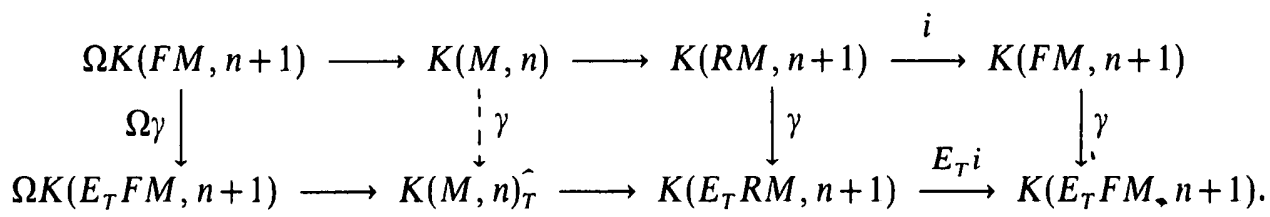

We have written $K(M, n)$ for the fibre of $i$ since it is such an Eilenberg-MacLane $G$-space by inspection of the fibrations obtained by passage to fixed point spaces. Define $K(M, n)_{T}$ to be the fibre of $E_{T} i$. By (8) and the long exact homotopy sequences of the fixed point space fibrations,

$$
\pi_{n} K(M, n)_{T}^{\hat{n}}=E_{T} M \quad \text { and } \quad \pi_{n+1} K(M, n)_{T}^{\hat{~}}=H_{T} M .
$$

Thus, by criterion (i) of Theorem $2, K(M, n)_{T}$ is $T$-complete. The right square commutes by functoriality, hence there exists a unique dotted arrow $\gamma$ such that the diagram commutes. (This is unnecessarily precise; all we need is that the diagram commutes up to $G$-homotopy.) Checking criterion (ii) of Theorem 3 on fixed point spaces by use of the $\bmod p$ homology Serre spectral sequences for $p \in T$, we see that $\gamma: K(M, n) \rightarrow K(M, n)_{T}$ is a completion at $T$.

To complete the proof of Theorem 14, we may assume that our $G$-nilpotent $G$-space is given as a nilpotent $G$-tower, and we adopt the notations of [5, Definition 6].

Construction 15. Let $X$ be a nilpotent $G$-tower. Starting with $\left(X_{0}\right)_{T}=\{*\}$, construct compatible completions $\gamma_{n}: X_{n} \rightarrow\left(X_{n}\right)_{T}$ inductively by means of diagrams

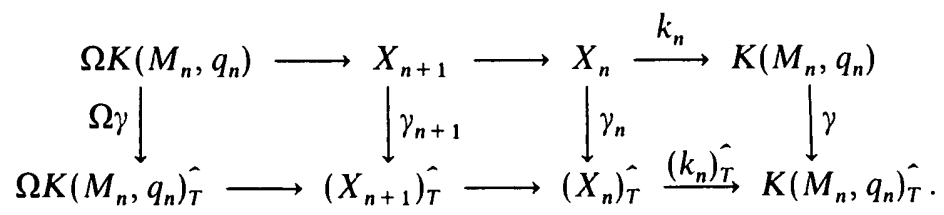


Here $\left(X_{n+1}\right)_{T}$ is defined to be the fibre of $\left(k_{n}\right)_{T}$, and $\gamma_{n+1}$ is obtained by the standard Barratt-Puppe sequence construction. The details are exactly as in [5, Construction 12], and we obtain a completion $\gamma: X \rightarrow \hat{X}_{T}$ on passage to limits.

The moral is that there is no reason to restrict to Eilenberg-MacLane spaces as the building blocks of towers (or, dually, to spheres as the building blocks of cell complexes). See [4] for a general discussion.

Remark 16. For finite $G$, the semi-simplicial construction of Bousfield and Kan [1] automatically generalizes to a functorial (but not continuously functorial) construction of equivariant completions.

Remark 17. For finite $G$, Hopf $G$-spaces $K(A, G)$ and $K O(A, G)$ representing the equivariant algebraic $K$-theory and orthogonal algebraic $K$-theory of a ring $A$ were introduced in [3]. For the algebraic closure $\bar{k}_{q}$ of the field with $q$ elements, Brauer lift Hopf G-maps

$$
\beta: K\left(\bar{k}_{q}, G\right) \rightarrow K(G) \text { and } \beta: K O\left(\bar{k}_{q}, G\right) \rightarrow K O(G)
$$

were constructed and shown to be $\hat{T}$-cohomology isomorphisms, where $T$ is the set of primes other than $q$ and $K(G)$ and $K O(G)$ represent complex and real equivariant $K$-theory. As in the nonequivariant case, the domains here are not of finite type and these maps are not $T$-cohomology isomorphisms. On passage to $G$-connected covers and then to completions at $T$, the maps $\beta$ become equivalences. Use of the orthogonal case equivalence is a necessary ingredient in the argument for the identification of $K O\left(k_{r}, G\right)$ as the fibre of $\psi^{r}-1: K O(G) \rightarrow K O(G)$ sketched at the end of [3], where $k_{r}$ is the field of $r=q^{a}$ elements and $q$ is prime to the order of $G$.

\section{References}

1. A. K. Bousfield and D. M. KAN, Homotopy limits, completions, and localizations, Lecture Notes in Mathematics 304 (Springer, Berlin, 1972).

2. A. Elmendorf, 'Equivariant homotopy and fixed point sets', Trans. Amer. Math. Soc., to appear.

3. Z. Fiedorowicz, H. HAUSChILD and J. P. MAY, 'Equivariant algebraic $K$-theory', Proceedings of the Oberwolfach, 1980 , conference on algebraic $K$-theory. Springer Lecture Notes in Mathematics, to appear.

4. J. P. MAY, The homotopical foundations of algebraic topology (Academic Press), in preparation.

5. J. P. MaY, J. MCClure and G. Triantafillou, 'Equivariant localization', this.Bulletin, pp. 223-230.

6. D. Sullivan, 'The genetics of homotopy theory and the Adams conjecture', Ann. of Math. (2), 100 (1974), 1-79.

Department of Mathematics, University of Chicago,

Chicago,

Illinois 60637,

U.S.A. 